\title{
Séance de préparation de transparents nécessaires aux exposés oraux en anglais
}

\section{Laurence Peyraud}

\section{(2) OpenEdition}

Journals

Édition électronique

URL : http://journals.openedition.org/asp/4385

DOI : 10.4000/asp.4385

ISSN : 2108-6354

Éditeur

Groupe d'étude et de recherche en anglais de spécialité

Édition imprimée

Date de publication : 1 mars 1993

Pagination : 367-370

ISSN : 1246-8185

Référence électronique

Laurence Peyraud, « Séance de préparation de transparents nécessaires aux exposés oraux en anglais », ASp [En ligne], 1 | 1993, mis en ligne le 09 mai 2014, consulté le 19 avril 2019. URL : http:// journals.openedition.org/asp/4385; DOI : 10.4000/asp.4385

Ce document a été généré automatiquement le 19 avril 2019

Tous droits réservés 


\title{
Séance de préparation de transparents nécessaires aux exposés oraux en anglais
}

\author{
Laurence Peyraud
}

1 Comment aider les étudiants à préparer ces transparents ? Pour beaucoup d'entre eux, c'est la première fois qu'ils utilisent des transparents. Ainsi je consacre une séance en début d'année, pour expliquer le but des transparents et créer une série de transparents nécessaires à l'accompagnement d'un exposé oral.

\section{Matériel nécessaire}

2 Une photocopie par étudiant d'un article tiré de Vocable avec les explications de vocabulaire, le même article pour tous; quatre feuilles de papier blanc par groupe de deux étudiants ; des feutres de couleurs. Par souci d'économie, nous utilisons des feuilles blanches et pas de vrais transparents à ce stade, car il s'agit de l'élaboration de transparents qui ne serviront pas véritablement à un exposé, ce n'est qu'un entraînement.

\section{Déroulement de la séance}

3 Explication générale de l'utilité des transparents et présentation de quelques exemples (10 min) ; distribution de l'article à chaque étudiant; lecture silencieuse de l'article (15 min); discussion et partage du travail entre les deux étudiants qui travaillent ensemble (10 $\mathrm{min})$.

4 Exécution des quatre transparents, un pour le titre et le plan de l'article, un pour les mots-clés de vocabulaire et deux pour l'illustration à proprement parée du contenu de 
l'article (45 min); affichage des transparents sur les murs de la salle et évaluation individuelle des transparents de chaque groupe (10 $\mathrm{min})$.

\section{Discussion}

5 Après plusieurs années d'expérience de cette séance, je peux annoncer que le déroulement de celle-ci est très régulier, compte tenu du sujet et de la longueur de l'article en fonction du niveau de compétence langagière des étudiants, bien entendu.

6 Les étudiants prennent conscience du travail de création et d'interprétation demandé au moment de l'illustration des données du texte. Les données chiffrées sont facilement traduites en tableaux ou graphiques, mais les symboles, les idées abstraites sont autant d'éléments que chaque étudiant va représenter selon sa sensibilité, sa culture ou son sens de l'humour. Les étudiants sont donc motivés par la confection de ces transparents. Le fait de travailler par deux pendant cette préparation les stimule et leur donne le sens du partage des tâches.

7 Au moment de l'affichage et de l'évaluation, selon des critères choisis ensemble, les étudiants perçoivent les différences d'interprétations des données et les styles de présentation. Cette comparaison entre les différents travaux leur permet de toucher du doigt les possibilités de cet outil que sont les transparents.

Enfin, ce n'est qu'à la première présentation orale individuelle qui a lieu quatre semaines plus tard, que chaque étudiant applique vraiment les directives et les conseils enregistrés pendant cette séance.

D'autre part en écoutant et suivant les divers exposés il se rend compte du rapport qui existe entre le contenu de la présentation orale et l'aide visuelle. Il comprend l'impact que peuvent avoir les transparents sur le public et il apprend à jouer de la complémentarité de ceux-ci par rapport au discours. Il prend aussi conscience de l'aisance et de la liberté dont le présentateur peut jouir pour parler sans lire ses notes s'il utilise les transparents d'une façon appropriée.

10 Ainsi les étudiants faibles en anglais peuvent s'exprimer plus facilement grâce aux transparents, car l'effet de diversion que ceux-ci produisent diminue son état de malaise. Pour conclure, je dirai que les transparents sont utilisables pour toutes sortes de catégories de présentations orales : résumés d'articles; exposés techniques; exercices de rhétorique ; sujets libres.

\section{ANNEXES}

\section{Annexe : questions de l'auditoire}

La séance se tient-elle en anglais? Oui, sauf pendant l'élaboration des transparents où les étudiants les plus faibles échangent leurs idées en français. 
Comment faites-vous pour qu'il n'y ait pas trop d'informations sur les transparents ? On leur annonce que la clarté est essentielle et ensuite ils l'apprennent par eux-mêmes, à leurs dépens.

S'ils utilisaient de vrais transparents dès le début, serait-ce un avantage ? Peut-être que ce serait mieux en effet, ils feraient plus attention aux consignes et ils verraient le résultat d'une façon immédiate.

Comment s'organise cette séance parmi les quatre consacrées aux exposés oraux dans votre cours? Sur 75 heures annuelles de cours d'anglais à la MIAG d'Orsay, les étudiants passent quatre séances de $1 \mathrm{~h} 30$ à la présentation orale. La première consiste à l'élaboration de transparents en vue de se préparer aux trois exposés oraux exigés dans cette formation.

\section{AUTEUR}

\section{LAURENCE PEYRAUD}

Enseignante d'anglais à la Maîtrise d'Informatique Appliquée à la Gestion (MlAG) de Paris-Sud, Orsay. laurence.peyraud@u-psud.fr 